\title{
Usefulness of Submental Ultrasonographic Evaluation for Dysphagia Patients
}

\author{
Yoon Soo Lee, MD, Ko Eun Lee, MD, Yeon Kang, MD, Tae Im Yi, MD, Joo Sup Kim, MD, PhD
}

Department of Rehabilitation Medicine, Bundang Jesaeng General Hospital, Seongnam, Korea

\begin{abstract}
Objective To investigate the usefulness of ultrasonographic measurement of hyoid bone movement during swallowing.

Methods Fifty-two patients who had swallowing dysfunction were enrolled in this study. When a patient swallowed $5 \mathrm{~mL}$ of water while maintaining an upright sitting position, hyoid bone movement during swallowing was measured with ultrasonography. Recorded images were analyzed to measure the maximum change in hyoid bone displacement. Mandible was used as reference point to calculate hyoid bone displacement. The farthest distance from resting position and the nearest distance during swallowing were measured and their differences were recorded. Participants also underwent videofluoroscopic swallowing study (VFSS). Based on penetrationaspiration scale (PAS), they were grouped to non-aspirators (PAS 1), penetrators (PAS 2-5), or aspirators (PAS 6-8). Measured hyoid bone displacements by submental ultrasonography were compared among groups.

Results The mean hyoid bone displacement in non-aspirators group $(\mathrm{n}=21,15.9 \pm 2.7 \mathrm{~mm})$ was significantly $(\mathrm{p}<0.05)$ greater than that in penetrators group $(\mathrm{n}=20,11.5 \pm 2.8 \mathrm{~mm})$ or aspirators group $(\mathrm{n}=11,8.0 \pm 1.0 \mathrm{~mm})$. Hyoid bone displacement below $13.5 \mathrm{~mm}$ as a cutoff point for detecting penetration or aspiration had a sensitivity and specificity of $83.9 \%$ and $81.0 \%$, respectively.

Conclusion Submental ultrasonographic evaluation was well correlated with PAS measured by VFSS. Therefore, submental ultrasonographic evaluation could be a useful screening tool for dysphagic patients.
\end{abstract}

Keywords Ultrasonography, Deglutition disorders, Deglutition, Hyoid bone

\section{INTRODUCTION}

Hyoid bone movement plays an important role in opening the upper esophageal sphincter by causing epiglottis

Received May 29, 2015; Accepted August 20, 2015

Corresponding author: Ko Eun Lee

Department of Rehabilitation Medicine, Bundang Jesaeng General Hospital, 20 Seohyeon-ro 180beon-gil, Bundang-gu, Seongnam 13590, Korea

Tel: +82-31-779-0063, Fax: +82-31-779-0635, E-mail: rhsrhs2u@gmail.com

(c) This is an open-access article distributed under the terms of the Creative Commons Attribution Non-Commercial License (http://creativecommons. org/licenses/by-nc/4.0) which permits unrestricted noncommercial use, distribution, and reproduction in any medium, provided the original work is properly cited.

Copyright $\odot 2016$ by Korean Academy of Rehabilitation Medicine tilting. When there is a pathological decrease or delay in such a process, it becomes the main cause of aspiration $[1,2]$. Examiner may perform palpation to assess laryngeal elevation using hand, a subjective, non-quantitative method. Videofluoroscopic swallowing study (VFSS) can provide relatively accurate measurements. However, if patient cannot reach the examination system, the study cannot be performed. In addition, VFSS has a drawback of radiation exposure that may occur during the examination $[3,4]$.

Hsiao et al. [5] introduced a method to measure hyoid bone displacement during swallowing by using ultraso- 
nography in 2012. This method showed excellent intraexaminer and inter-examiner reliabilities. It has been demonstrated that there were significant differences in hyoid bone displacement during swallowing between a tube feeding group and an oral intake group based on functional oral intake scale (FOIS). However, the FOIS is a scale based on current diet type of patients [6]. It has limitation in reflecting clinical seriousness.

Therefore, hyoid bone displacement during swallowing was measured in patients with dysphagia in this study by performing submental ultrasonographic evaluation. The aim of this study was to identify the usefulness of submental ultrasonographic evaluation for dysphagia patients by analyzing the correlation between penetration-aspiration scale (PAS) and pharyngeal residue grade to identify the correlation between hyoid bone displacement and degree of dysphagia.

\section{MATERIALS AND METHODS}

\section{Subjects}

This study was performed in patients with dysphagia as the main complaint. They underwent VFSS. Those who were admitted to the Department of Rehabilitation Medicine, Bundang Jesaeng General Hospital, or patients were referred to the Department of Rehabilitation Medicine for consultation from June 2014 to August 2014. These subjects were categorized into three groups according to PAS while swallowing $5 \mathrm{~mL}$ of liquid during VFSS. Results were compared by classifying residues in pyriform sinus and vallecular fossa into four levels after swallowing $5 \mathrm{~mL}$ of liquid. PAS was categorized into 1 to 8 levels depending on the level of the airway up to which the food passed during swallowing, whether it entered the airway, and whether any effort was made by the patient to eject the aspirated food out of the airway. When material did not enter the airway, it was considered as PAS 1 . When material entered the airway and remained above the vocal folds followed by ejection from the airway, it was considered as PAS 2 . When material entered the airway and remained above the vocal folds without being ejected from the airway, it was considered as PAS 3 . When material entered the airway and contacted the vocal folds followed by ejection from the airway, it was considered as PAS 4. When material entered the airway and contacted the vocal folds without being ejected from the airway, it was considered as PAS 5. When material entered the airway and passed below the vocal folds followed by ejection into the larynx or out of the airway, it was considered as PAS 6. When material entered the airway and passed below the vocal folds without being ejected from the trachea despite effort, it was rated as PAS 7 . When material entered the airway and passed below the vocal folds without effort of ejection, it was rated as PAS 8 [7]. In this study, the non-aspirators group was defined as PAS 1 , the penetrators group was defined as PAS 2-5, and the aspirators group was defined as PAS 6-8. Depending on the amount of residues in pyriform sinus and vallecular fossa after swallowing as shown in the lateral images of VFSS, the grades were defined. Grade 0 was considered when there was no residue based on the height of an applicable structure. Grade 1 was defined when the residue was less than $10 \%$ of the structure height. Grade 2 was considered when the residue was more than $10 \%$ but less than $50 \%$ of the structure height. Grade 3 was defined when the residue was $\geq 50 \%$ of the structure height [8].

\section{Methods}

Hyoid bone displacement in patients was measured by performing submental ultrasonographic evaluation on the same day after VFSS was performed. After a vertical line was drawn towards the mandible from the superior thyroid notch, an ultrasound probe was placed along the line in the longitudinal direction (Fig. 1). The examination was conducted with patient sitting in the upright position while the neck was maintained in a neutral position. Patient was allowed to swallow $5 \mathrm{~mL}$ of liquid mixed with contrast media of the same viscosity as that used in VFSS. During the examination, the mandible was pressed slightly to ensure that the probe was immobile during swallowing. The swallowing was then induced by the patient. The examination was performed by measuring the distance between the shades created from the mandible and the hyoid bone at rest and during swallowing (Fig. 2). The difference in the distance was defined as hyoid bone displacement. The percentage of hyoid bone displacement for the distance between the shades at rest was defined by delta value. In this study, an ultrasonographic diagnostic equipment LOGIQ E9 (GE Healthcare, Milwaukee, WI, USA) with a curved probe of 1-5 MHz was used. 

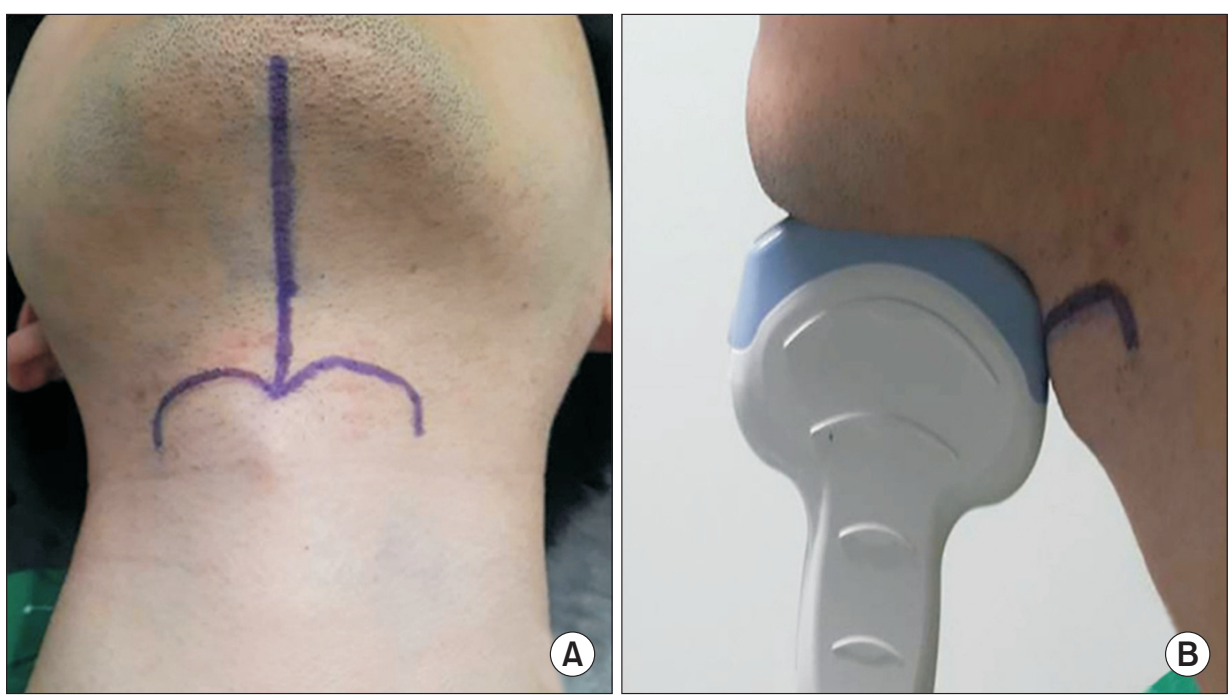

Fig. 1. Submental ultrasonographic evaluation. (A) A vertical line is drawn from superior thyroid notch to mandible for probe placement. (B) Probe was placed at midsagittal plane of submental area.
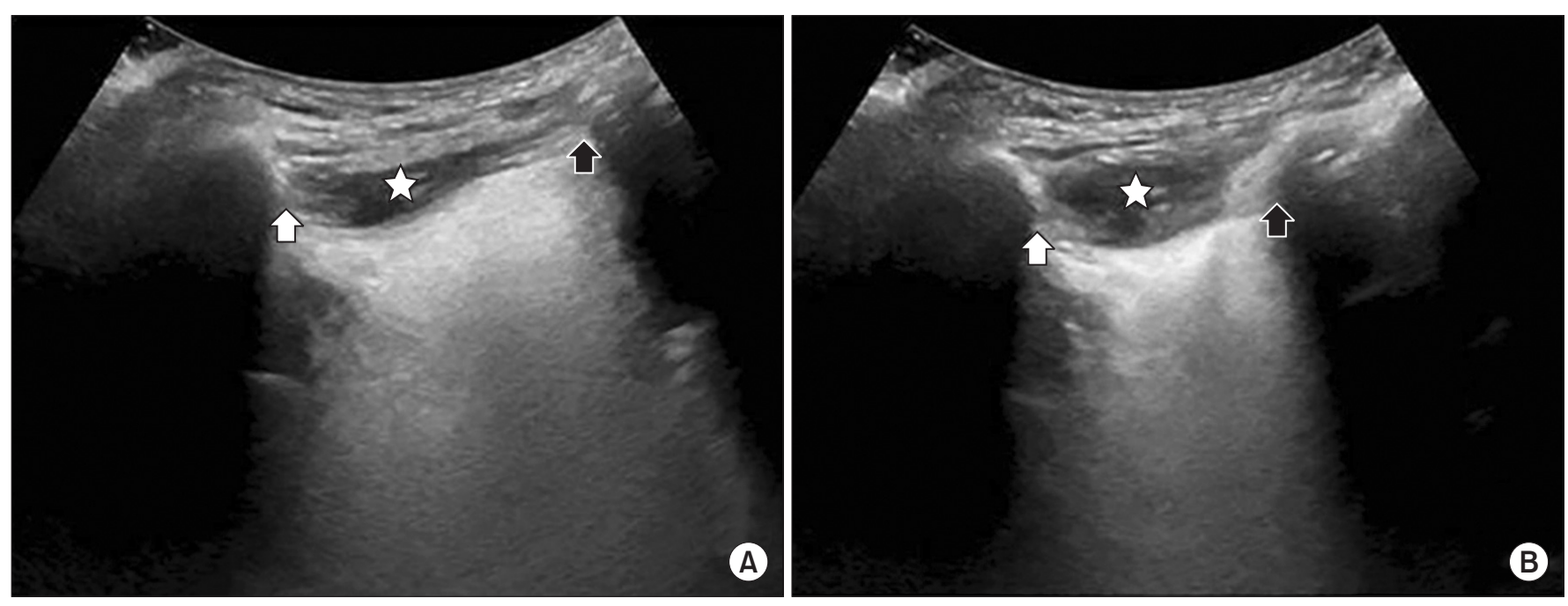

Fig. 2. Hyoid bone displacement. (A) Distance between the mandible and the hyoid bone at rest, (B) during swallowing. White arrow, shadow behind the mandible; black arrow, shadow behind the hyoid bone; asterisk, suprahyoid muscles.

\section{Statistical analyses}

Kruskal-Wallis test, a nonparametric rank sum test, was used to analyze the verification of the inter-group differences in hyoid bone displacement within groups that were categorized according to PAS and post-swallowing residue in the pyriform sinus or vallecular fossa. The result of a post-hoc test between the two subgroups within each respective group was analyzed using Mann-Whitney $\mathrm{U}$ test. In order to determine the cutoff value of hyoid bone displacement and delta value to predict the presence/absence of penetration-aspiration according to PAS, the point at which the sum of sensitivity and speci- ficity reached its maximum at the apex of the ROC curve with the farthest coordinates of the vertical distance from the baseline was set as the cutoff value. SPSS ver. 18.0 (SPSS Inc., Chicago, IL, USA) for Windows was used for statistical analysis. Statistical significance was considered when $p$ value $\leq 0.05$.

\section{RESULTS}

\section{General characteristics of subjects}

The general characteristics of subjects included in this study are summarized in Table 1 . The mean age of sub- 
Yoon Soo Lee, et al.

Table 1. General characteristics of subjects

\begin{tabular}{lccc}
\hline & $\begin{array}{c}\text { Non-aspirators } \\
(\mathbf{n = 2 1 )}\end{array}$ & $\begin{array}{c}\text { Penetrators } \\
(\mathbf{n = 2 0})\end{array}$ & $\begin{array}{c}\text { Aspirators } \\
(\mathbf{n}=\mathbf{1 1})\end{array}$ \\
\hline Age & $64.1 \pm 13.4$ & $61.5 \pm 15.9$ & $55.1 \pm 21.6$ \\
& $(58.0-70.2)$ & $(54.0-69.0)$ & $(40.6-69.7)$ \\
Sex (male:female) & $13: 8$ & $14: 6$ & $7: 4$ \\
Diagnosis & & & \\
$\quad$ Stroke (hemorrhage: infarction) & $10: 8$ & $7: 11$ & $5: 4$ \\
$\quad$ Others & 3 & 2 & 2 \\
\hline
\end{tabular}

Values are presented as mean \pm standard deviation (range) or number.

Table 2. Relationship between hyoid bone displacement and penetration-aspiration scale

\begin{tabular}{lcccc}
\hline & $\begin{array}{c}\text { Non-aspirators } \\
(\mathbf{n = 2 1})\end{array}$ & $\begin{array}{c}\text { Penetrators } \\
(\mathbf{n = 2 0})\end{array}$ & $\begin{array}{c}\text { Aspirators } \\
(\mathbf{n}=\mathbf{1 1})\end{array}$ & p-value \\
\hline Resting distance $(\mathrm{mm})$ & $39.3 \pm 5.4$ & $37.8 \pm 2.4$ & $37.2 \pm 4.0$ & \\
Shortest distance $(\mathrm{mm})$ & $24.4 \pm 4.9$ & $26.2 \pm 2.9$ & $29.4 \pm 4.1$ & \\
Hyoid bone displacement $(\mathrm{mm})_{\text {Delta value }^{\text {a) }}(\%)}^{15.9 \pm 2.7}$ & $11.5 \pm 2.8$ & $8.0 \pm 1.0$ & $<0.001^{*}$ \\
\hline
\end{tabular}

Values are presented as mean \pm standard deviation.

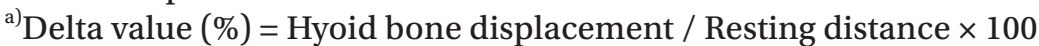

${ }^{*} \mathrm{p} \leq 0.05$ statistically significant (Kruskal-Wallis test).

jects was $61.2 \pm 16.4$ years. There were 34 men and 18 women among the total 52 subjects. In this study, subjects were assigned to three groups according to the PAS based on VFSS. There were 21 subjects in the nonaspirators group, 20 subjects in the penetrators group, and 11 subjects in the aspirators group. There were no statistically significant ( $p>0.05)$ differences in age or gender distribution of patients among the three groups. The cognitive function did not show statistically significant difference either among the three groups. These patients mostly suffered from stroke. The number of patients who suffered from other conditions such as brain tumor, Parkinson disease, and poor oral intake in addition to stroke was 3 in the non-aspirators group, 2 in the penetrators group, and 2 in the aspirators group.

Association between hyoid bone displacement and penetration-aspiration scale

Association results between hyoid bone displacement and PAS are summarized in Table 2. The mean value of hyoid bone displacement was smaller in the group with higher PAS score. The differences in mean value of hyoid bone displacement among groups were statistically significant $(p<0.001)$. Based on post-hoc test, hyoid bone displacement distance was significantly $(\mathrm{p}<0.001)$ shorter in both the penetrators group and the aspirators group compared to that in the non-aspirators group, while the displacement distance in the aspirators group was significantly $(\mathrm{p}=0.001)$ shorter than that in the penetrators group. Delta values were smaller in groups with greater PAS scores. The differences in delta values among groups were statistically significant $(p<0.001)$. Based on post-hoc test results, delta values in the penetrators group $(\mathrm{p}=0.001)$ and the aspirators group $(\mathrm{p}<0.001)$ were smaller than that in the non-aspirators group. In addition, the delta value in the aspirators group was also significantly $(\mathrm{p}=0.002)$ smaller than that in the penetrators group. When based on hyoid bone displacement distance as $13.5 \mathrm{~mm}$, the cutoff value of hyoid bone displacement according to PAS showed a sensitivity of $83.9 \%$ and specificity of $81.0 \%$. When based on delta value as $30.3 \%$, the cutoff delta value according to PAS showed a sensitivity of $64.5 \%$ and a specificity of $95.2 \%$.

Association between hyoid bone displacement and post-swallowing pharyngeal residue grade

Association results between hyoid bone displacement and the degree of post-swallowing residue in the pyri- 
Table 3. Relationship between hyoid bone displacement and pyriform sinus residue

\begin{tabular}{lcccc}
\hline & $\begin{array}{c}\text { Grade 0 } \\
(\mathbf{n = 1 5 )}\end{array}$ & $\begin{array}{c}\text { Grade 1 } \\
(\mathbf{n = 2 5})\end{array}$ & $\begin{array}{c}\text { Grade 2-3 } \\
(\mathbf{n = 1 2})\end{array}$ & p-value \\
\hline Resting distance $(\mathrm{mm})$ & $39.0 \pm 4.2$ & $37.7 \pm 3.0$ & $38.9 \pm 4.8$ & \\
Shortest distance (mm) & $25.1 \pm 5.5$ & $25.3 \pm 2.9$ & $29.5 \pm 4.3$ & \\
Hyoid bone displacement $(\mathrm{mm})_{\text {Delta value }^{\text {a) }}(\%)}^{14.8 \pm 3.6}$ & $12.5 \pm 3.2$ & $9.4 \pm 3.8$ & $0.001^{*}$ \\
\hline
\end{tabular}

Values are presented as mean \pm standard deviation.

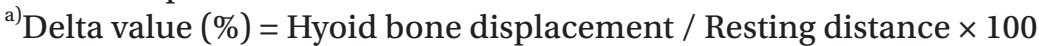

${ }^{*} \mathrm{p} \leq 0.05$ statistically significant (Kruskal-Wallis test).

Table 4. Relationship between hyoid bone displacement and vallecular fossa residue

\begin{tabular}{lcccc}
\hline & $\begin{array}{c}\text { Grade 0 } \\
(\mathbf{n = 1 1 )}\end{array}$ & $\begin{array}{c}\text { Grade 1 } \\
(\mathbf{n = 2 4})\end{array}$ & $\begin{array}{c}\text { Grade 2-3 } \\
(\mathbf{n = 1 7})\end{array}$ & p-value \\
\hline Resting distance $(\mathrm{mm})$ & $39.6 \pm 4.3$ & $38.3 \pm 3.7$ & $37.6 \pm 3.6$ & \\
Shortest distance $(\mathrm{mm})$ & $25.0 \pm 6.0$ & $25.6 \pm 3.9$ & $27.9 \pm 3.7$ & \\
Hyoid bone displacement $(\mathrm{mm})_{\text {Delta value }^{\text {a) }} \text { (\%) }}^{15.9 \pm 3.5}$ & $12.9 \pm 3.5$ & $9.7 \pm 2.7$ & $<0.001^{*}$ \\
\hline
\end{tabular}

Values are presented as mean \pm standard deviation.

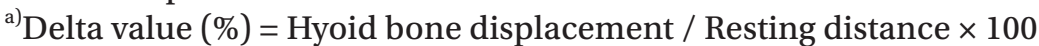

${ }^{*} \mathrm{p} \leq 0.05$ statistically significant (Kruskal-Wallis test).

form sinus are shown in Table 3. There were 15 patients in post-swallowing pharyngeal residue grade 0 group, 25 patients in grade 1 group, 6 patients in grade 2 group, and 6 patients in grade 3 group. The number of patients in grade 2 and grade 3 groups was smaller compared to that in grade 0 or grade 1 group. Therefore, statistical analysis was conducted by combining grade 2 group and grade 3 group into one group. The mean value of hyoid bone displacement in the group that had more post-swallowing residues in the pyriform sinus was significantly $(\mathrm{p}=0.001)$ smaller compared to that in other groups. Based on results of post-hoc test, the mean value of hyoid bone displacement showed statistically significant difference between grade 0 group and grade $2-3$ group $(\mathrm{p}=0.001)$ as well as between grade 1 group and grade $2-3$ group $(\mathrm{p}=0.004)$. The delta value in the group with a larger residue in the pyriform sinus after swallowing was significantly $(\mathrm{p}=0.001)$ smaller. Based on post-hoc test results, the delta value showed statistically significant differences between grade 0 group and grade $2-3$ group $(\mathrm{p}<0.001)$ as well as between grade 1 group and grade $2-3$ group $(\mathrm{p}=0.001)$.

The association results between hyoid bone displacement and the grade of post-swallowing residue in the val- lecular fossa are shown in Table 4. There were 11 patients in the group of grade 0,24 patients in grade 1 group, 13 patients in grade 2 group, and 4 patients in grade 3 group. As the number of patients in grade 3 group was very small, statistical analysis was conducted by pooling grade 2 group and grade 3 group into one group. The mean value of hyoid bone displacement was smaller in the group with more post-swallowing residue in the vallecular fossa. Differences among the groups were statistically significant $(\mathrm{p}<0.001)$. Based on post-hoc test results, the mean values of hyoid bone displacement showed statistically significant differences between grade 0 group and grade 1 group $(\mathrm{p}=0.036)$, between grade 0 group and grade $2-3$ group $(p<0.001)$, and between grade 1 group and grade $2-3$ group $(p=0.005)$. Delta value was smaller in the group with larger grade of post-swallowing residue in the vallecular fossa. Differences among groups were statistically significant ( $\mathrm{p}=0.001)$. Based on post-hoc test results, delta values were significantly different between grade 0 group and grade $2-3$ group $(p<0.001)$ as well as between grade 1 group and grade $2-3$ group $(\mathrm{p}=0.005)$. 


\section{DISCUSSION}

Early treatment of dysphagia has been known to be able to reduce medical problems caused by aspiration pneumonia, malnutrition, and dehydration with reduced length of hospital stay $[9,10]$. Therefore, accurate evaluation of dysphagia in the early stage is important for successful treatment of dysphagia. Dodds et al. [11] have reported that only visual examination of swallowing in a patient cannot clearly reveal dysphagia in the pharyngeal phase. Unlike the widely accepted opinions, the swallowing ability in the pharyngeal phase has no correlation with complete gag reflex. Several bedside tests have been introduced to evaluate dysphagia, including the utilization of cough reflex, 3-ounce water test, and laryngeal elevation. DePippo et al. [12] have suggested that bedside tests were valuable for diagnosing conditions such as aspiration pneumonia or airway obstruction. In 2003, Yoon et al. [13] evaluated the significance of performing these bedside tests as dysphagia screening tests in patients who complained of dysphagia or were suspected of having dysphagia, but not in patients with aspiration pneumonia. They performed the study to evaluate the usefulness of adding gag reflex and laryngeal elevation. Dysphagia bedside test showed a certain degree of sensitivity, indicating that it had some value as a screening test. However, it showed less negative predictability and specificity. For this reason, swallowing function should be evaluated objectively through radiologic examination [13].

VFSS, an objective evaluation of swallowing, is advantageous as it is an absolute standard to make a usual diagnosis of dysphagia. However, it is also disadvantageous as the examination cannot be carried out if the patient cannot reach where the examination equipment is located. In addition, the patient may be exposed to radiation during the examination. Ultrasonographic evaluation for dysphagia is another objective testing method with merits since it is relatively easier to apply without the need of moving the patient to a special examination room. In addition, it can be applied to bedridden patients without exposing patients to radiation [5]. Shawker et al. [14] have used ultrasonographic examination due to its benefits of being non-invasive. They were able perform real-time verification in order to visualize tongue movements in 8 healthy subjects and in patients with neurological damage accompanied by dysphagia as well as chronic aspira- tion. Hsiao et al. [5] have suggested that submental ultrasonography can be used to visualize bolus transporting in the oral stage as a reliable measurement for hyoid bone displacement in the pharyngeal phase. Therefore, it can aid bedside assessment of swallowing function among stroke patients.

Hyoid bone has been advantageously used as an anatomical index of the laryngeal movement due to radiopacity. It is an important anatomical point for analyzing swallowing in most of methodological studies for the evaluation of VFSS [15-17]. Hyoid bone and larynx show anterior-superior displacement caused by the contraction of suprahyoid muscle during swallowing. Superior displacement of the hyoid bone is highly variable. It can be influenced by the initial consistency of food, whereas anterior displacement of the hyoid bone is less variable. It is associated with opening of the upper esophageal sphincter [18]. Anterior displacement of the hyoid bone occurs simultaneously with anterior displacement of the entire larynx caused by contraction of the geniohyoid muscle, thus contributing to the generation of negative pressure in the upper esophageal sphincter. The negative pressure generated in the esophageal sphincter plays a role not only in suction that helps downward movement of the food, but also in preventing aspiration by influencing laryngeal obstruction caused by vocal cord adduction and epiglottis flip located at the split edge of the airway and the esophagus [19]. Hence, reduced hyoid bone displacement suggests the possibility of dysphagia which can interfere with smooth downward movement of the bolus, causing aspiration or abnormal opening of the upper esophageal sphincter. Hsiao et al. [5] have reported that hyoid bone displacement might be used as an additional predictor of tube feeding dependency. However, Steele et al. [20] have reported that there would be more pharyngeal residue in patients with less anterior displacement of the hyoid bone.

This study was conducted to investigate the kind of association between hyoid bone movement during swallowing and PAS, a clinical scale of VFSS. The distance between shades created by the mandible and the hyoid bone at rest (Fig. 2A) was measured through submental ultrasonographic evaluation. The distance between shades newly created by the mandible and the hyoid bone due to anterior-superior movement of the hyoid bone during swallowing (Fig. 2B) was also measured. The 
difference between these two measurements was defined as hyoid bone displacement. The aim of this study was to measure the degree of contraction of the geniohyoid muscle among the suprahyoid muscles and to identify the extent of association with symptoms of dysphagia seen in pharyngeal and laryngeal phases due to hyoid bone movement in connection with laryngeal elevation and opening of the upper esophageal sphincter. According to our results, the group with a higher score of PAS had significantly smaller hyoid bone displacement and smaller percentage of hyoid bone displacement for the distance from the mandible to the hyoid bone, i.e., the delta value (Table 2). This indicates that when dysphagia in the pharyngeal phase is severe in VFSS, it has a tendency of having a smaller hyoid bone displacement. The reason why the group with a higher PAS score has significantly smaller hyoid bone displacement could be due to the fact that the hyoid bone displacement was too small to induce appropriate laryngeal elevation or to contribute to proper negative pressure formation in the upper esophageal sphincter. Therefore, the risk of aspiration is increased.

Hsiao et al. [5] have set the cutoff value of hyoid bone displacement based on $15.0 \mathrm{~mm}$ in their study in order to classify tube feeding group and oral intake group according to FOIS. That cutoff value showed a sensitivity of $73.3 \%$ and a specificity of $66.7 \%$. However, in this study, considering that each person has a different anatomic structure such as the position of the hyoid bone and the total length of the neck, the cutoff delta value according to PAS was calculated by defining the percentage of hyoid bone displacement for the distance between the shades created from the mandible and the hyoid bone at rest as the delta values. The cutoff value of hyoid bone displacement that can predict the presence/absence of penetration-aspiration according to the PAS showed a sensitivity of $83.9 \%$ and a specificity of $81.0 \%$ when it was set at 13.5 $\mathrm{mm}$. These results implied that $83.9 \%$ of patients who had hyoid bone displacement less than $13.5 \mathrm{~mm}$ belonged to the penetrators group and the aspirators group, whereas $81.0 \%$ of patients who had hyoid bone displacement at $13.5 \mathrm{~mm}$ or higher belonged to the groups with neither penetration or aspiration. In addition, when the cutoff delta value according to PAS was set at $30.3 \%$. It showed a sensitivity of $64.5 \%$ and a specificity of $95.2 \%$. These results implied that $64.5 \%$ of patients who had delta value less than $30.3 \%$ belonged to the penetrators group and the aspirators group, whereas $95.2 \%$ of patients whose delta value was $30.3 \%$ or higher belonged to the groups with neither penetration nor aspiration. The fact that this study showed higher levels of sensitivity and specificity than the study by Hsiao et al. [5] may indicate that the results of VFSS-based PAS reflected clinical seriousness more sensitively since FOIS scale based on dietary pattern of patients. However, since this study did not include a sufficient number of patients, further study with more number of patients is needed in the future. Sufficient validation of the cutoff value may be required to have clinical significance.

Post-swallowing pharyngeal residue is an important risk factor for the occurrence of aspiration [21]. Perlman et al. [22] have reported that epiglottis adduction does not occur in patients with residue in the vallecular fossa compared to patients do not have residue in the vallecular fossa. Even if adduction occurred, it was incomplete. They also reported that the incidence rate of oral involvement was higher and the degree of hyoid bone elevation was reduced in patients with dysfunctional epiglottis than those in patients without dysfunctional epiglottis. In this study, hyoid bone displacement during swallowing was measured by submental ultrasonographic evaluation in patients with dysphagia and post-swallowing residues in the pyriform sinus and vallecular fossa. According to our results, hyoid bone displacement was found to be lesser in the group with larger amount of residue in the pyriform sinus and the vallecular fossa (Tables 3, 4). In other words, in VFSS, it was confirmed that the group with a larger amount of food residues remaining in the pyriform sinus and vallecular fossa after swallowing tended to have significantly lesser absolute and relative displacement of the hyoid bone. This indicated that the hyoid bone displacement measurement could be a predictable factor for post-swallowing pharyngeal residue level. The association between reduced hyoid bone displacement and post-swallowing pharyngeal residue level could be due to the fact that abnormal opening of the upper esophageal sphincter was induced that the inner pressure could not be formed properly during swallowing, thus increasing post-swallowing pharyngeal residue level.

There are some limitations of this study. First, the number of patients in the group with relatively severe aspi- 
ration or with a large amount of residue was too small, making it difficult to verify the statistical significance. There were 11 patients in the aspirators group applicable to PAS 6-8, 6 patients in grade 2 group with the amount of post-swallowing residue in the pyriform sinus from the lateral images of VFSS $\geq 10 \%$ and $<50 \%$ of the applicable structural height, and 6 patients in grade 3 group with the amount of post-swallowing residue in the pyriform sinus $\geq 50 \%$. There were 4 patients in grade 3 group with the amount of residue in the vallecular fossa $\geq 50 \%$. These numbers were comparatively small. They might have affected the verification of statistical significance. Therefore, statistical analysis was conducted by incorporating grade 2 group and grade 3 group into one group. Second, because VFSS and submental ultrasonographic evaluation were not performed simultaneously in this study during one time swallowing, we could not rule out the possibility that the outcomes of these two tests might have been affected by differences in the testing time points. Nevertheless, since the two tests were performed on the same day, the swallowing function of patients was less likely to affect the test outcomes as notable changes between the two tests. Third, this study was performed collectively without classifying the causal diseases of dysphagia or patients with stroke based on the location of the lesion due to the limited number of patients. In the future, additional studies should be conducted with larger sample sizes. Additional confirmation should also be obtained on whether hyoid bone displacement showed any differences according to the causal disease of dysphagia and the lesion location in stroke patients. It is also necessary to make a comparison with non-disability group without dysphagia. Fourth, this study was conducted by using only $5 \mathrm{~mL}$ of liquid without diversifying the viscosity of food. Hence, additional studies are needed to further verify whether there is any difference in hyoid bone displacement according to the viscosity of food.

In conclusion, our results revealed that hyoid bone displacement measured through submental ultrasonographic evaluation showed statistically significant correlation with PAS, a clinical scale of VFSS and pharyngeal residue grade. Therefore, dysphagia evaluation performed by submental ultrasonographic evaluation can be used to determine swallowing function of patients in the pharyngeal phase in a relatively easier manner. This is beneficial as a screening test for dysphagia pa- tients for early detection and treatment for patients who need dysphagia therapy in clinical aspects. It also has its merits as patients will not be exposed to radiation during the examination. In addition, the swallowing function of patients at bedside in the pharyngeal phase can be identified in a comparatively easier manner for those patients who cannot reach the place where VFSS equipment is installed. Therefore, it can be used for such patients for treatment of dysphagia and follow-up evaluation accordingly by assessing the improvement in swallowing function during the clinical process of dysphagia therapy, thus providing feedback to patients.

\section{CONFLICT OF INTEREST}

No potential conflict of interest relevant to this article was reported.

\section{REFERENCES}

1. Ishida R, Palmer JB, Hiiemae KM. Hyoid motion during swallowing: factors affecting forward and upward displacement. Dysphagia 2002;17:262-72.

2. Logemann JA, Kahrilas PJ, Cheng J, Pauloski BR, Gibbons PJ, Rademaker AW, et al. Closure mechanisms of laryngeal vestibule during swallow. Am J Physiol 1992;262(2 Pt 1):G338-44.

3. Martino R, Pron G, Diamant N. Screening for oropharyngeal dysphagia in stroke: insufficient evidence for guidelines. Dysphagia 2000;15:19-30.

4. Yabunaka K, Sanada H, Sanada S, Konishi H, Hashimoto T, Yatake H, et al. Sonographic assessment of hyoid bone movement during swallowing: a study of normal adults with advancing age. Radiol Phys Technol 2011;4:73-7.

5. Hsiao MY, Chang YC, Chen WS, Chang HY, Wang TG. Application of ultrasonography in assessing oropharyngeal dysphagia in stroke patients. Ultrasound Med Biol 2012;38:1522-8.

6. Crary MA, Mann GD, Groher ME. Initial psychometric assessment of a functional oral intake scale for dysphagia in stroke patients. Arch Phys Med Rehabil 2005;86:151620.

7. Rosenbek JC, Robbins JA, Roecker EB, Coyle JL, Wood JL. A penetration-aspiration scale. Dysphagia 1996;11:93-8.

8. Han TR, Paik NJ, Park JW. Quantifying swallowing function after stroke: a functional dysphagia scale based 
on videofluoroscopic studies. Arch Phys Med Rehabil 2001;82:677-82.

9. Elmståhl S, Bülow M, Ekberg O, Petersson M, Tegner H. Treatment of dysphagia improves nutritional conditions in stroke patients. Dysphagia 1999;14:61-6.

10. Poertner LC, Coleman RF. Swallowing therapy in adults. Otolaryngol Clin North Am 1998;31:561-79.

11. Dodds WJ, Logemann JA, Stewart ET. Radiologic assessment of abnormal oral and pharyngeal phases of swallowing. AJR Am J Roentgenol 1990;154:965-74.

12. DePippo KL, Holas MA, Reding MJ. The Burke dysphagia screening test: validation of its use in patients with stroke. Arch Phys Med Rehabil 1994;75:1284-6.

13. Yoon K, Kim SB, Lee KW, Park SH. Clinical utility of the bedside swallowing evaluations for dysphagia. J Korean Acad Rehabil Med 2003;27:489-93.

14. Shawker TH, Sonies B, Stone M, Baum BJ. Real-time ultrasound visualization of tongue movement during swallowing. J Clin Ultrasound 1983;11:485-90.

15. Kellen PM, Becker DL, Reinhardt JM, Van Daele DJ. Computer-assisted assessment of hyoid bone motion from videofluoroscopic swallow studies. Dysphagia 2010;25:298-306.

16. Dengel G, Robbins J, Rosenbek JC. Image processing in swallowing and speech research. Dysphagia 1991;6:30-9.
17. Logemann JA, Kahrilas PJ, Begelman J, Dodds WJ, Pauloski BR. Interactive computer program for biomechanical analysis of videoradiographic studies of swallowing. AJR Am J Roentgenol 1989;153:277-80.

18. Braddom RL, Chan L, Harrast HA, Kowalske KJ, Matthews DJ, Ragnarsson KT, et al. Physical medicine and rehabilitation. 4th ed. Philadelphia: Saunders; 2011. p. 581600.

19. Yokoyama M, Mitomi N, Tetsuka K, Tayama N, Niimi S. Role of laryngeal movement and effect of aging on swallowing pressure in the pharynx and upper esophageal sphincter. Laryngoscope 2000;110(3 Pt 1):434-9.

20. Steele CM, Bailey GL, Chau T, Molfenter SM, Oshalla M, Waito AA, et al. The relationship between hyoid and laryngeal displacement and swallowing impairment. Clin Otolaryngol 2011;36:30-6.

21. Eisenhuber E, Schima W, Schober E, Pokieser P, Stadler A, Scharitzer M, et al. Videofluoroscopic assessment of patients with dysphagia: pharyngeal retention is a predictive factor for aspiration. AJR Am J Roentgenol 2002; 178:393-8.

22. Perlman AL, Grayhack JP, Booth BM. The relationship of vallecular residue to oral involvement, reduced hyoid elevation, and epiglottic function. J Speech Hear Res 1992; 35:734-41. 